\title{
Novel compound heterozygous COG5 mutations in a Chinese male patient with severe clinical symptoms and type IIi congenital disorder of glycosylation: A case report
}

\author{
SHAOWEI YIN ${ }^{1-3}$, LIYING GONG $^{4}, \mathrm{HAO}^{5} \mathrm{QIU}^{5}$, YAN ZHAO $^{6}$, YAN ZHANG ${ }^{7}$, CAIXIA LIU $^{1-3}$, \\ HONGKUN JIANG ${ }^{8}$, YAN MAO ${ }^{5}$, LING-YIN KONG ${ }^{5}$, BO LIANG ${ }^{9}$ and YUAN LV ${ }^{1-3}$ \\ ${ }^{1}$ Liaoning Centre for Prenatal Diagnosis, Key Laboratory of Maternal-Fetal Medicine of Liaoning Province; \\ ${ }^{2}$ Key Laboratory of Obstetrics and Gynecology of Higher Education of Liaoning Province; \\ ${ }^{3}$ Department of Obstetrics and Gynecology, Shengjing Hospital Affiliated to China Medical University, Shenyang, \\ Liaoning 110004; ${ }^{4}$ Clinical Laboratory, The People's Hospital of China Medical University, Shenyang, Liaoning 110016; \\ ${ }^{5}$ Basecare Medical Device Co., Ltd., Suzhou, Jiangsu 215000; ${ }^{6}$ Genetics Unit; ${ }^{7}$ The Outpatient Department of \\ Obstetrics, Shenyang Maternity and Infant's Hospital, Shenyang, Liaoning 110004; ${ }^{8}$ Department of Pediatrics, \\ The First Affiliated Hospital of China Medical University, Shenyang, Liaoning 110016; ${ }^{9}$ State Key Laboratory \\ of Microbial Metabolism, Joint International Research Laboratory of Metabolic and Developmental Sciences, \\ School of Life Sciences and Biotechnology, Shanghai Jiao Tong University, Shanghai 200240, P.R. China
}

Received November 30, 2018; Accepted July 5, 2019

DOI: $10.3892 / \mathrm{etm} .2019 .7834$

\begin{abstract}
In the current study, one case of COG5-CDG involving a Chinese male patient with severe neurological symptoms, who had previously been misdiagnosed with congenital gyrus malformation, is described. A clinical investigation was performed and targeted next-generation sequencing (NGS) was used to identify COG5 variants in the patient and his family. PCR and Sanger sequencing were performed for the verification of NGS results. The patient showed severe central and peripheral neurological symptoms, while only mild symptoms were reported in a previous reported case, in which different mutations were involved. The reported patient carried the frameshift mutation c.330delT (p.V111Lfs $\left.{ }^{*} 22\right)$, and a missense mutation c.2324 C>T (p.P775L) in the COG5 gene. The c.330delT (p.V111Lfs'22) variant is a novel mutation, while c. $2324 \mathrm{C}>\mathrm{T}$ (p.P775L) has previously been reported. Inheriting one variant from each of his parents, the current case report furthers the understanding of genotype-phenotype correlations in COG5-CDG.
\end{abstract}

Correspondence to: Dr Yuan Lv, Department of Obstetrics and Gynecology, Shengjing Hospital Affiliated to China Medical University, 36 Sanhao Street, Shenyang, Liaoning 110004, P.R. China E-mail: hawk.lv@163.com

Key words: congenital disorders of glycosylation, conserved oligomeric golgi 5, compound heterozygous mutations, severe symptoms

\section{Introduction}

Mutations in subunit five of the conserved oligomeric Golgi (COG) complex gives rise to type IIi congenital disorders of glycosylation (CDG) (1). CDG is physiologically related to other pathologies involving glycan defects and can lead to multiple organ failure, dysmorphism, skeletal malformation, hormonal disorders and coagulopathy (2). A total of eight subunits, which are associated with the retrograde transport of Golgi components, are present in the COG complex). Mutations in the COG1, COG2, COG4, COG5, COG6, COG7, and COG8 genes have been reported to cause CDG (COG-CDG), which can result in heterogeneous clinical phenotypes ranging from severe multi-organ disorders to moderate forms of neurological impairment $(3,4)$.

Only 10 COG5-CDG cases have been reported worldwide (2,5-8). Common symptoms among these cases include neurological, morphological and hepatic abnormalities $(7,8)$. Defects in the COG5 protein are mainly associated with abnormalities of the brain, liver, bladder and auditory and visual systems (9). Fung et al (5) described the first Chinese patient with COG5-CDG. In this aforementioned study, a female presented with mild neurohepatic disease and central and peripheral neurological involvement.

\section{Case report}

In the current study, a case of COG5-CDG involved a male Chinese patient with severe symptoms. To the best of our knowledge, this is the second COG5-CDG case to be reported in China. The 11-year-old patient (Fig. 1B) was the second child of a Chinese couple. During the gestation period, a TORCH examination was performed and the 
A
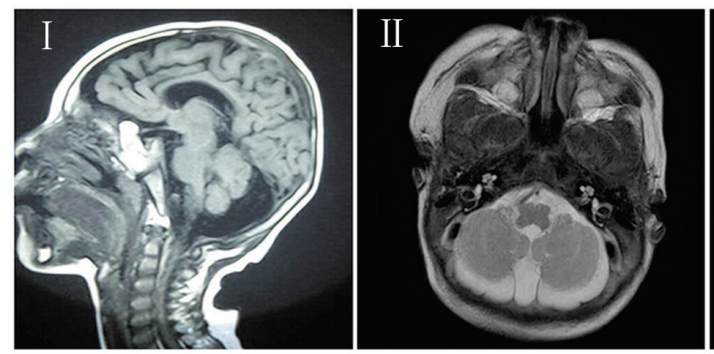

B

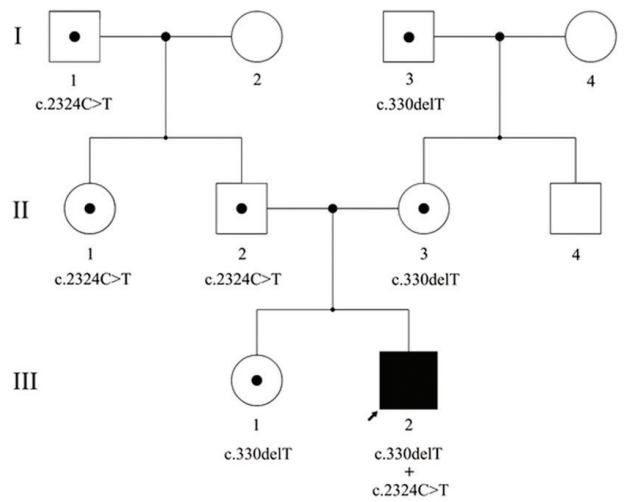

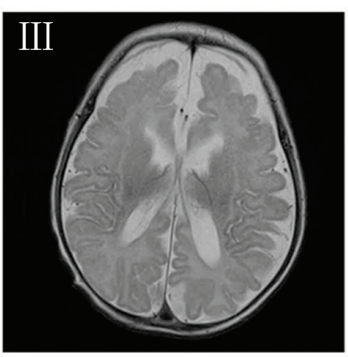

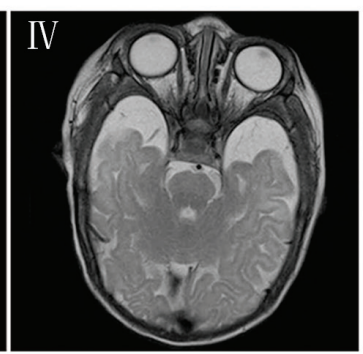

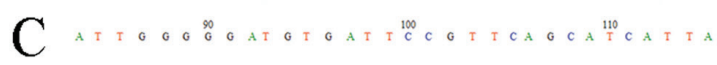
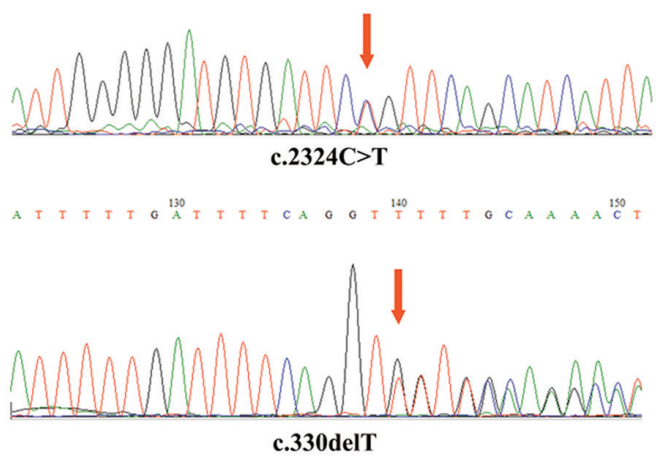

$\mathrm{D}$

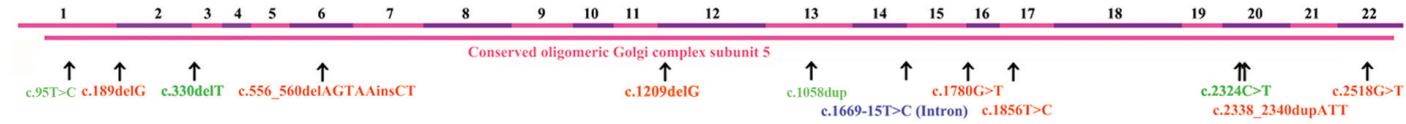

E

$\begin{array}{lc}\text { Human } & \begin{array}{c}\text { COG5 Protei } \\ \text { Mouse }\end{array} \\ \text { Chimp } & 829 \mathrm{aa} \\ \text { Chicken } & 860 \mathrm{aa} \\ \text { Sheep } & 941 \mathrm{aa} \\ \text { Zebra finch } & 858 \mathrm{aa} \\ \text { Platypus } & 817 \mathrm{aa} \\ \text { Rabbit } & 828 \mathrm{aa} \\ \text { Dog } & 841 \mathrm{aa} \\ \text { Marmoset } & 996 \mathrm{a} \\ & 823 \mathrm{aa}\end{array}$

p.V111Lfs*22 (In exon 3) VVARHEDLLAQATGIESLEG VVARHEDLLAQATGIESLEG VVARHEDLLAQATGIESLEG VVARHEDLLAQATGIESLEG VVARHEDLLAQATGIESLEG VVARHEDLLAQATGIESLEG VVTRHEDLLAQATGIESLEG VVARHEDLLAQATGIESLEG VVARHEDLLAQATGIESLEG VVARHEDLLAQATGIESLEG
p.775P $>$ L (In exon 20)

PLLFQASEHVASSPALGDVIPFSIIIQFLFTRAPAELKSPFQ PLLFQTSEHVADSPAVGDIIPFSIIIQFLFTRAPAELKSPFQ PLLFQASEHVANSPALGDVIPFSIIIQFLFTRAPAELKSPFQ PLLFQTSEHIASSPALGEVIPFSIILQFLFTRAPPELKSPFQ PLLFQTSEHVASSPALGDIIPFSIIIQFLFTRAPPELKSPFQ PLLFQTSEHIASSPALGEVIPFSIILQFLFTRAPPELKSPFQ PLLFQTSEHVAASPALGEVIPFSTVLQLLFTRAPPELKSPFQ PLLFQTSEHVANSPALGDIIPFSVVIQFLFTRAPAELKSPFQ PLLFQTSEHVASSPALGDIIPFSIIIQFLFTRAPAELKSPFQ PLLFQTSEHVANSPALGDVIPFSIIIQFLFTRAPAELKSPFQ

Figure 1. MRI results and novel mutations in a patient with COG5-CDG. (A) MRI of the patient case. (B) The pedigree of the patient's family. (C) COG5 mutations detected in the male patient (III-2) are indicated by arrows. (D) Schematic of previously reported COG5 mutations and the two mutations present in the case patient. (E) Conservation of COG5 residues. COG5, conserved oligomeric golgi 5; CDG, congenital disorders of glycosylation.

results were negative, and ultrasound results were normal except for polyhydramnios, which was noted in the second trimester. The patient was born at full-term via caesarean section. Upon physical examination, the patient's head and feet were slightly smaller than average, the small forehead and an umbilical hernia was discovered. An MRI of the brain at 1.5 months revealed brain hypotrophy and thinning of the corpus callosum. At 6.5 months, the patient exhibited slow responses, glassy eyes, and appeared epileptic with the presence of mild microcephaly. The patient feet were still slightly smaller than average, and the upper limbs appeared hypermyotonic. The patient was unable to turn over, crawl, or grasp objects. An additional MRI was performed at 12 months and revealed cerebellar atrophy (Fig. 1A). Fig. 1A-I and 1A-II images revealed cerebellar hemisphere and vermis hypoplasia at midst sagittal plane and cerebellum level respectively, while Fig. 1A-III revealed the bilateral temporal lobes and Fig. 1A-IV showed frontal lobes hypogenesis. The patients' symptoms were still present at 18 months and the patient was unable walk and, even when holding onto a desk, could only stand for short periods of time. Speech development was absent, although according to the parents, the patient was able to understand a few words. All other family members presented a normal phenotype (Table I).

Total genomic DNA was extracted according to standard protocols (TIANamp Genomic DNA kit; Tiangen Biotech Co., Ltd.) from peripheral blood leukocytes isolated from the patient and his family members. Targeted enrichment of the whole exome DNA was performed using the Nextera Rapid Capture Exome kit (Illumina, Inc.) according to manufacturer's protocol. Whole exome libraries were then sequenced on an Illumina HiSeq platform (Illumina, Inc.) using a $2 \times 100 \mathrm{bp}$ sequencing protocol (10). The results indicated the presence of potentially homozygous, compound heterozygous, and de novo variants, which were selected for further investigation. Variants with minor allele frequencies $<0.01$ in any of the dbSNP, ExAC, 1000 Genomes Project, gnomAD, or in-house databases were selected for further exploration. 


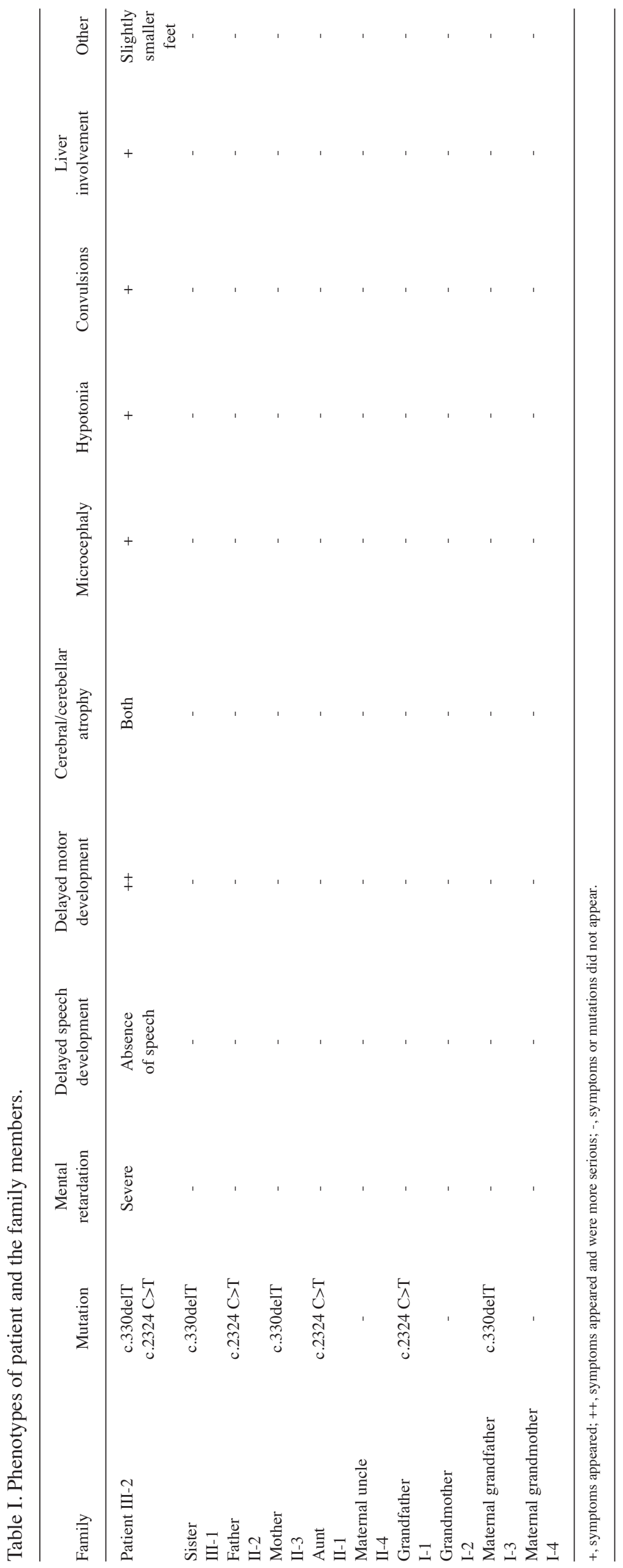




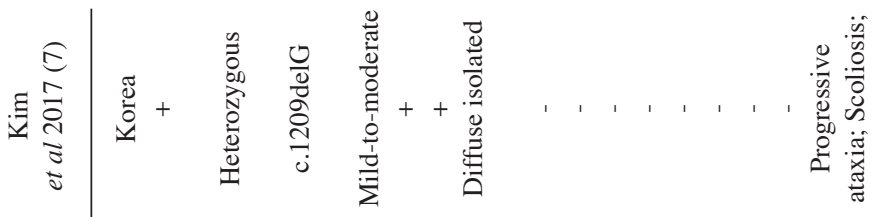

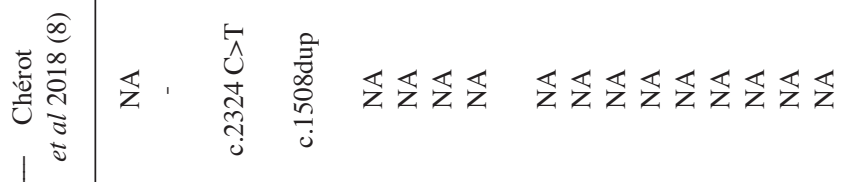

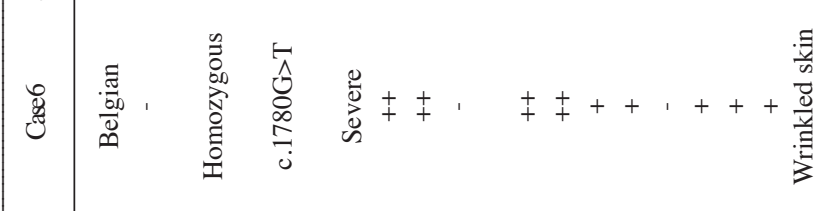

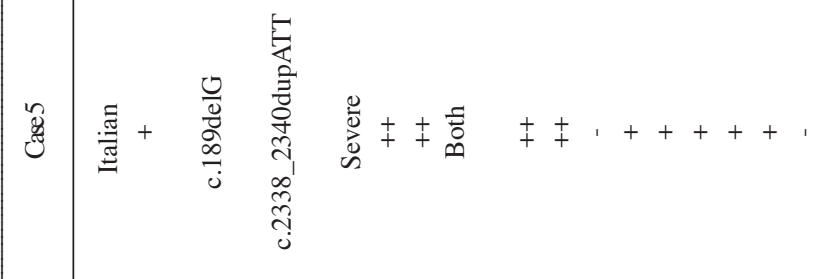

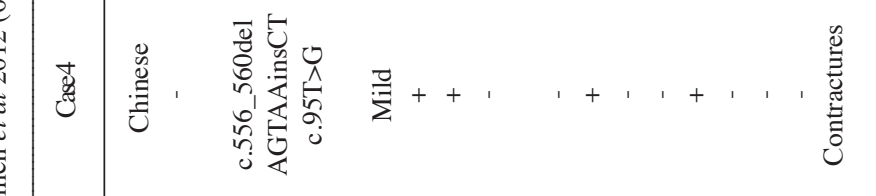

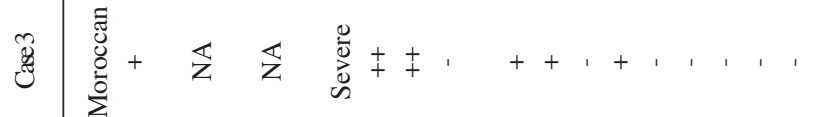

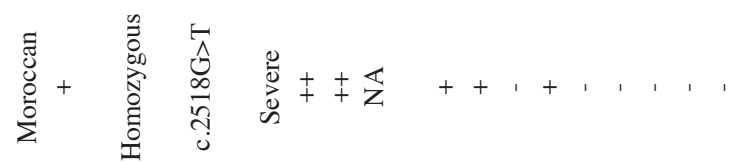

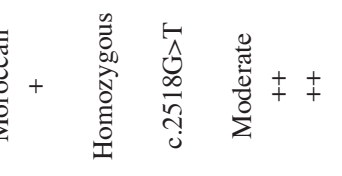

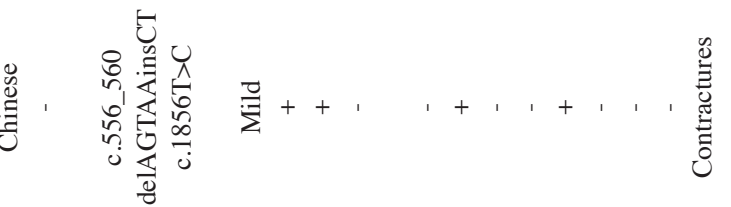

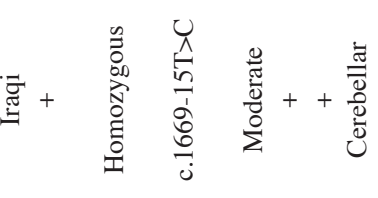

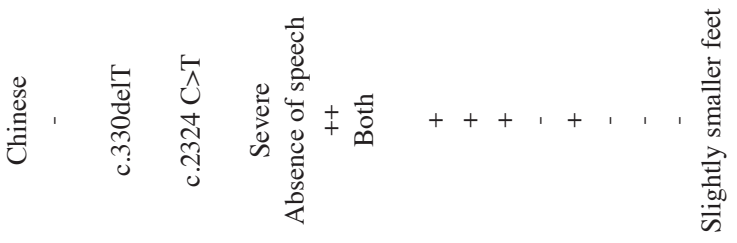


After data filtration, a potential compound heterozygous mutation(NM_006348.3: c.330delT,p.V111Lfs*22; c.2324C>T, p.P775L) was identified. Then the c.330delT mutation was amplified using a specific forward (5'-TCCCGCTTACAACTC ATCT-3') and reverse primer (5'-CCAATGGGGAAGTCGAT GCT-3'), while c.2324C $>$ T mutation was amplified by specific primer pair: Forward 5'-TTCTCCCACCAAATCACCTA-3' and reverse 5'-TGCTCTCGTGAACAAAAACTG-3'. PCR was conducted with Premix Taq (TaKaRa Taq Version 2.0; R004A). The primer extension was cycled for 35 cycles with $15 \mathrm{sec}$ at $95^{\circ} \mathrm{C}, 15 \mathrm{sec}$ at $53^{\circ} \mathrm{C}(\mathrm{c} .330 \mathrm{delT}) / 55^{\circ} \mathrm{C}(\mathrm{c} .2324 \mathrm{C}>\mathrm{T})$, and $15 \mathrm{sec}$ at $72^{\circ} \mathrm{C}$. Following PCR amplification, Sanger sequencing confirmed that NM_006348.3: c.2324C $>$ T was inherited paternally and that I-1 and II-1 were carriers of this mutation (Fig. 1B and C). NM_006348.3: c.330delT was inherited maternally, while I-3 and III-1 were carriers of this variant (Fig. 1B and C). Therefore, the results revealed that the patient carried a frameshift mutation, c.330delT (p.V111Lfs*22), and a missense mutation, c.2324 C>T (p.P775L), in the COG5 gene. Due to both variants involving the same transcript (NM_006348.3), the presence of a compound heterozygous mutation was observed.

To the best of our knowledge, in the 1000 Genomes project (http://www.internationalgenome.org/), the Exome Aggregation Consortium (http://exac.broadinstitute.org/), the gnomAD (http://gnomad-old.broadinstitute.org/) and our in-house database, neither variant had previously been described. The NM_006348.3: c.2324 C>T, p.P775L mutation was recently reported to be pathogenic (8). This was supported by the American College of Medical Genetics (ACMG) guidelines, which classified this variant as pathogenic (categories, PM2, PM3, PP3 and PP5) (11). This mutation is highly conserved among a variety of species (Fig. 1E; https://genome. ucsc.edu/). The prediction, regarding the effect of this mutation, with SIFT (PROVEAN v1.1.3) and PolyPhen-2 (version 2.2) software indicated that the protein variant was 'deleterious' and 'probably damaging', respectively. The NM_006348.3: c.330delT variant (p.V111Lfs ${ }^{*} 22$ ) is a frameshift mutation that leads to the production of an aberrant and truncated protein of 132 amino acids. This frameshift variant may result in nonsense-mediated decay, leading to a premature termination codon (PTC) at exon 4 (22 exons in total). According to the ACMG guidelines, this frameshift mutation was placed into the PVS1 and PM2 categories, thereby classifying it as a likely pathogenic variant (11).

\section{Discussion}

Defects of the COG complex indirectly affect glycosylation through the altered trafficking of glycosyltransferases (4). Therefore, the identification of a variety of forms of CDG, which are caused by COG deficiency, founded a new understanding of CDG pathogenesis. The COG complex is composed of eight subunits (named COG1-8), which are separated into two sub-complexes: Lobe A (COGs 1-4) and lobe B (COGs $5-8)(12,13)$. It has been suggested that comparable molecular defects may be more detrimental in lobe A COG-CDG than in lobe B COG-CDG (12).

Including the present case, twelve COG5 mutations have been identified worldwide (Table II). Most of these mutations
(9/12) are located in exons, while one mutation is a homozygous intronic substitution that leads to COG5 exon skipping (Fig. 1D). Due to the COG5 protein being highly conserved among numerous species (Fig. 1E), the full-length protein is important for its function, while lobe A appears to be essential for maintaining overall Golgi structure and lobe B impacts recognition and vesicle tethering to the right Golgi compartment (14). In the current case, the c.330delT frameshift mutation led to a patched (PTC) of COG5 protein translation, which generated a truncated 133-amino acid protein. According to the mechanism of action, the location of the PTC may lead to nonsense mediated decay. This hypothesis could not be tested in the current study due to a limited sample quantity. However, it is possible that this mutation may cause a loss of function in COG5. The c.2324C $>\mathrm{T}$ missense mutation causes an amino acid substitution at residue 755 , from proline to leucine.

The c. $2324 \mathrm{C}>\mathrm{T}$ mutation was detected in the patient's father. Having received two different COG5 mutations from each parent, a compound heterozygous mutation was therefore observed in this case. Abnormalities in glycoconjugate synthesis, intracellular protein sorting and protein secretion have been revealed to be caused by COG defects. COG serves an important role in the regulation, compartmentalization or activity of multiple Golgi glycosylation enzymes $(15,16)$. As a subunit of COG, although less severe, COG5 deficiency in HeLa cells causes the dilation of a number of Golgi cisternae, defects in global glycosylation and damage to intracellular processing (1). Additionally, MALDI TOF analysis revealed hypoglycosylation and the lack of a terminal sialic acid of serum transferrin in patients with COG5-CDG $(5,6)$. Therefore, it is suggested that the novel COG5 compound heterozygous mutations may lead to glycosylation defects in the proband, resulting in COG5-related CDG, but this needs to be investigated.

The COG5-8 gene exhibits a relative tolerance to loss of function variants (12), and its phenotypic severity is in line with the tolerance to other COG gene variants. COG5 mutations have been indicated to lead to relatively mild phenotypes. In the current case report, the patient exhibited phenotypes of mental retardation, absence of speech, delayed motor development, cerebral and cerebellar atrophy, microcephaly, hypotonia, convulsions, liver involvement and slightly smaller feet. Compared with lobe A, the patient exhibited a milder phenotype. However, in comparison with the previously reported COG5-CDG case (6-8), the patient showed severe central and peripheral neurological symptoms.

In conclusion, a case of COG5-CDG in a Chinese patient is reported, in which relatively severe clinical symptoms were present. Further investigation into the relationship between the phenotype and genotype of COG5-CDG will facilitate a better understanding of this rare disease.

\section{Acknowledgements}

Not applicable.

\section{Funding}

The present study was jointly supported by grants from the Natural Science Foundation of China (grant no. 81701462) 
and the Obstetric Diseases Translational Medicine Research Center Project of Liaoning Province (grant no. 2014225007).

\section{Availability of data and materials}

All data generated or analyzed during the present study are included in this published article.

\section{Authors' contributions}

SWY, LYG and YL contributed to the conception, design and performed experiments. YL and HQ contributed to the analysis of the data, and the drafting and revising of the manuscript.. YZhao, YZhang, CXL, HKJ and YL were responsible for clinical diagnosis. YM, LYK and BL contributed to the acquisition of the data and data analysis and interpretation. All authors are accountable for all aspects of the work and read and approved the final version of the manuscript.

\section{Ethics approval and consent to participate}

All procedures performed in studies involving human participants were in accordance with the Ethical Standards of the Institutional and/or National Research Committee and in accordance with the 1964 Helsinki Declaration and its later amendments or comparable ethical standards. Informed consent was obtained from the legal guardians of the patient in this study.

\section{Patient consent to publication}

A written informed consent for the publication of the images was provided by the patient's guardians.

\section{Competing interests}

The authors declare that they have no conflicts of interest.

\section{References}

1. Oka T, Vasile E, Penman M, Novina CD, Dykxhoorn DM, Ungar D, Hughson FM and Krieger M: Genetic analysis of the subunit organization and function of the conserved oligomeric golgi (COG) complex: Studies of COG5- and COG7-deficient mammalian cells. J Biol Chem 280: 32736-32745, 2005.

2. Paesold-Burda P, Maag C, Troxler H, Foulquier F, Kleinert P, Schnabel S, Baumgartner M and Hennet T: Deficiency in COG5 causes a moderate form of congenital disorders of glycosylation. Hum Mol Genet 18: 4350-4356, 2009.
3. Ungar D, Oka T, Brittle EE, Vasile E, Lupashin VV, Chatterton JE, Heuser JE, Krieger $M$ and Waters MG: Characterization of a mammalian Golgi-localized protein complex, COG, that is required for normal Golgi morphology and function. J Cell Biol 157: 405-415, 2002.

4. Barone R, Fiumara A and Jaeken J: Congenital disorders of glycosylation with emphasis on cerebellar involvement. Semin Neurol 34: 357-366, 2014.

5. Fung CW, Matthijs G, Sturiale L, Garozzo D, Wong KY, Wong R, Wong V and Jaeken J: COG5-CDG with a mild neurohepatic presentation. JIMD Rep 3: 67-70, 2012.

6. Rymen D, Keldermans L, Race V, Régal L, Deconinck N, Dionisi-Vici C, Fung CW, Sturiale L, Rosnoblet C, Foulquier F, et al: COG5-CDG: Expanding the clinical spectrum. Orphanet J Rare Dis 7: 94, 2012.

7. Kim YO, Yun M, Jeong JH, Choi SM, Kim SK, Yoon W, Park C, Hong Y and Woo YJ: A mild form of COG5 defect showing early-childhood-onset Friedreich's-Ataxia-Like phenotypes with isolated cerebellar atrophy. J Korean Med Sci 32: 1885-1890, 2017.

8. Cherot E, Keren B, Dubourg C, Carré W, Fradin M,Lavillaureix A, Afenjar A, Burglen L, Whalen S, Charles P, et al: Using medical exome sequencing to identify the causes of neurodevelopmental disorders: Experience of 2 clinical units and 216 patients. Clin Genet 93: 567-576, 2018.

9. Jaeken J and Péanne R: What is new in CDG? J Inherit Metab Dis 40: 569-586, 2017.

10. Bronner IF, Quail MA, Turner DJ and Swerdlow H: Improved protocols for Illumina sequencing. Curr Protoc Hum Genet 80: 18.2.1-42, 2014.

11. Richards S, Aziz N, Bale S, Bick D, Das S, Gastier-Foster J, Grody WW, Hegde M, Lyon E, Spector E, et al: Standards and guidelines for the interpretation of sequence variants: A joint consensus recommendation of the American College of Medical Genetics and Genomics and the Association for Molecular Pathology. Genet Med 17: 405-424, 2015.

12. Haijes HA, Jaeken J, Foulquier F and van Hasselt PM: Hypothesis: Lobe A (COG1-4)-CDG causes a more severe phenotype than lobe B (COG5-8)-CDG. J Med Genet 55: 137-142, 2018.

13. Ungar D, Oka T, Vasile E, Krieger M and Hughson FM: Subunit architecture of the conserved oligomeric Golgi complex. J Biol Chem 280: 32729-32735, 2005.

14. Peanne R, Legrand D, Duvet S, Mir AM, Matthijs G, Rohrer J and Foulquier F: Differential effects of lobe A and lobe B of the Conserved Oligomeric Golgi complex on the stability of \{beta\}1,4-galactosyltransferase 1 and \{alpha\}2,6-sialyltransferase 1. Glycobiology 21: 864-876, 2011.

15. Kingsley DM, Kozarsky KF, Segal M and Krieger M: Three types of low density lipoprotein receptor-deficient mutant have pleiotropic defects in the synthesis of N-linked, O-linked, and lipid-linked carbohydrate chains. J Cell Biol 102: 1576-1585, 1986.

16. Reddy $\mathrm{P}$ and Krieger M: Isolation and characterization of an extragenic suppressor of the low-density lipoprotein receptor-deficient phenotype of a Chinese hamster ovary cell mutant. Mol Cell Biol 9: 4799-4806, 1989. 